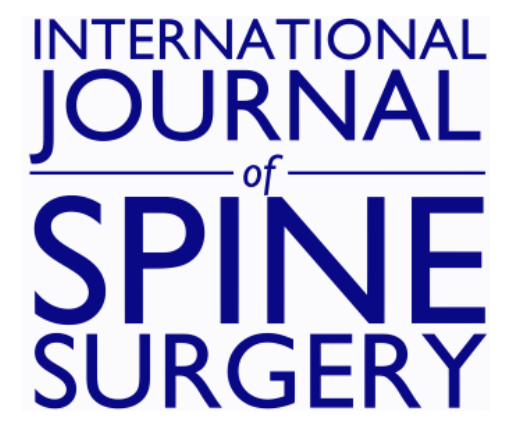

\title{
A systematic review of minimally invasive sacroiliac joint fusion utilizing a lateral transarticular technique
}

Jake Heiney, Robyn Capobianco and Daniel Cher

Int J Spine Surg 2015, 9 ()

doi: https://doi.org/10.14444/2040

http://ijssurgery.com/content/9/40

This information is current as of April 25, 2023.

Email Alerts Receive free email-alerts when new articles cite this article. Sign up at:

http://ijssurgery.com/alerts 


\section{A systematic review of minimally invasive sacroiliac joint fusion utilizing a lateral transarticular technique}

Jake Heiney, $M D,{ }^{1}$ Robyn Capobianco, $M A,^{2}$ Daniel Cher, $M D^{2}$

${ }^{1}$ University of Toledo Medical Center, Toledo, Ohio, ${ }^{2}$ SI-BONE, Inc., San Jose, California

\section{Abstract}

Background

A number of studies have been published regarding minimally invasive surgical (MIS) fusion of the sacroiliac (SI) joint using a lateral transarticular approach. Herein we report a systematic review and meta-analysis to summarize operative measures and clinical outcomes reported in published studies of MIS SI joint fusion.

\section{Methods}

The systematic review was done according to PRISMA standards. PubMed and EMBASE were searched using the terms sacroiliac joint AND fusion. Original peer-reviewed articles in the English language that reported clinical outcomes on at least 5 cases of MIS SI joint fusion using a lateral transarticular approach were included. Random effects meta-analysis (RMA) was performed on selected variables using the DerSimonian and Laird method, including operative measures, VAS SI joint pain ratings (0-10 scale) and Oswestry Disability Index (ODI). Mean and 95\% confidence intervals (CI) were calculated and heterogeneity was assessed. Other findings were summarized qualitatively.

Results

A total of 18 articles met the inclusion criteria. After accounting for overlapping cohorts, 12 unique cohorts from 4 countries were extracted for a total of 432 subjects. The RMA mean (range) was 59 minutes (27-78) for procedure time, $36.9 \mathrm{cc}$ (10-70) for estimated blood loss and 1.7 days (range 0-7) for length of stay (LOS). The RMA mean [95\% CI] pain score dropped by 5.2 points at 6 months and 5.3 points at 12 months (baseline score of 8.1 [7.8-8.4], 12-month score of 2.7 [2.1-3.3]), and a 24-month score of 2.0(1.4-2.5). ODI decreased by 31 points at 12 months (baseline score of 56.2 [51.0-61.5], 6-month score of 30.7 [21.8-39.6], and 12-month score of 25.1 [12.3-37.9]). Some estimates showed significant variation across studies and between the types of implants used. Other reported outcomes were supportive of the positive effects of SI joint fusion.

\section{Conclusion}

Published studies of MIS SI joint fusion using a lateral transarticular approach confirm its minimally invasive characteristics with minimal blood loss and short operating room times, and show consistent, rapid, sustained and clinically important improvements in patient reported SI joint pain, disability and quality of life scores.

KEYWORDS: MINIMALLY INVASIVE SURGERY, DEGENERATIVE SACROILIITIS, SACROILIAC JOINT DISRUPTION, SACROILIAC JOINT DYSFUNCTION, SACROILIAC JOINT FUSION, META-ANALYSIS, SYSTEMATIC REVIEW

VOLUME 9 ARTICLE 40 DOI: 10.14444/2040

\section{Background}

Low back pain (LBP) is a global health issue and one of the top 3 causes of health degradation in highly developed countries. ${ }^{1}$ In the United States, LBP is the second most common reason for visits to primary care physicians after the common cold. ${ }^{2}$ Annual expenditures for chronic back pain exceed $\$ 100$ billion per year in the U.S. alone. ${ }^{3}$ In addition to high levels of disability and lost income, LBP increases the risk of falls in the elderly, which can result in hip and/or spinal fractures subsequently increasing morbidity and mortality in this at risk population. ${ }^{4}$

While lumbar spinal structures are important factors to consider in patients who present with LBP, the sacroiliac (SI) joint is a well-known cause of pain in the lumbo-pelvic region that often presents in a manner similar to pathology arising from the lumbar spine or hip. The sacroiliac joint is the largest joint in 
the human body and is subject to shear forces of up to $4800 \mathrm{~N}$ with rotational movement of up to $4 \mathrm{de}-$ grees and translation capacity of $1.6 \mathrm{~mm} .{ }^{5}$ Studies in healthy volunteers have helped to validate the SI joint as a cause of pain and define its innervation. ${ }^{6,7}$ Substantial evidence suggests that the SI joint may be the pain generator in $15-30 \%$ of patients diagnosed with low back pain..$^{8-11}$ Etiologies of SI joint pain include degenerative sacroiliitis, inflammatory arthritis, SI joint disruptions from trauma or related to pregnancy, anatomical abnormalities such as leg length inequality and scoliosis, adjacent segment degeneration as a result of lumbar and lumbosacral spinal fixation procedures, infection, tumor, gout, and idiopathic causes. ${ }^{12,13}$

In patients who fail to improve after lumbar spinal arthrodesis, SI joint pain may explain the delayed onset of postoperative pain (indicating possible adjacent segment disease) or failure to improve as a result of possible misdiagnosis or presence of multiple pain generators. ${ }^{14-17}$ Radiographic evidence of SI degeneration can be seen in up to $75 \%$ of patients undergoing lumbar spinal fusion. ${ }^{18}$ Moreover, the SI joint was determined to be the pain generator in $43 \%$ of patients complaining of new onset or persistent pain after lumbar spinal fusion. ${ }^{19}$ Thus the SI joint not only plays an important role in low back pain, it also appears to be under-diagnosed.

The mainstay of care for sacroiliac pain is nonsurgical care. Non-surgical treatments for SI joint pain include pain medication, activity modification, weight loss, physical therapy, chiropractic care, radiofrequency ablation and sacroiliac joint steroid injections. ${ }^{10,20,21}$ However, there is only modest evidence that any of these non-surgical treatments are effective for any length of time. ${ }^{22}$ When these measures fail to provide significant and lasting symptom relief, surgical options may be considered.

Surgical treatment for persistent SI joint pain was first reported in the early $1900 \mathrm{~s} .{ }^{23}$ However, mainly due to challenges including blood loss, extended hospital stay, wound size and difficulty with mobilization (e.g. non-weight bearing status), open surgical repair is typically reserved for only the most severe cases. ${ }^{24,25}$ More recently, minimally invasive surgical
(MIS) methods have become available. Compared to open surgery, minimally invasive techniques are typically associated with less blood loss, shorter hospital stay, and less perioperative morbidity; these are likely attributable to smaller surgical incisions and less soft tissue dissection. Reports of MIS SI joint fusion confirm these benefits as well as show improved clinical outcomes, earlier post-operative weight bearing and overall improved patient satisfaction compared to open surgical techniques. ${ }^{26-28}$

To date, a number of studies have been published regarding minimally invasive fusion of the sacroiliac joint. The literature describes two significantly different approaches to achieve this goal: a dorsal joint distraction and a lateral transarticular approach. In the dorsal approach, the joint is distracted and a structural material (either implant, allograft) material is placed posterior to anterior within the joint. Using the lateral approach, implants are placed across the joint with the lateral portion of the implant residing in the ilium and the medial end in the sacrum. Thus the two techniques differ markedly with respect to the biomechanical stabilization of the SI, which is a necessary component of the arthrodesis procedure. The most common approach reported in the literature is the lateral transarticular approach. With this technique, the SI joint is accessed laterally through a small incision made into the buttocks. A drill is passed from the ilium into the sacrum and one or more implants are placed such that they span the joint from ilium to sacrum. The goal is to provide immediate fixation of the joint followed by a long-term arthrodesis. Cadaveric and finite element biomechanical studies ${ }^{29,30}$ have demonstrated marked reduction in SI joint motion after placement of transarticular implants. To our knowledge no biomechanical studies exist to support stabilization of the SI joint with a dorsal joint distraction technique.

Herein we present a systematic review with a quantitative meta-analysis (MA) of operative measures and clinical outcomes reported in published prospective or retrospective studies of MIS SI joint fusion using a lateral transarticular technique. 
Methods

We conducted a systematic review (SR) and metaanalysis (MA) according to PRISMA (Preferred Reporting Items for Systematic Reviews and MetaAnalyses) guidelines. ${ }^{31}$ PRISMA is a framework that provides a reproducible and transparent approach to reporting SRs and MAs. The databases Pubmed (http://www.ncbi.nlm.nih.gov/pubmed) and EMBASE (http://www.embase.com) were searched using the terms sacroiliac joint AND fusion. Pubmed is a publicly available database provided and maintained by the US National Library of Medicine at the $\mathrm{Na}$ tional Institutes of Health. EMBASE is a privately maintained database (Elsevier) available by institutional subscription and includes citations available in MEDLINE in addition to other journals not currently indexed in Pubmed. In addition to database reviews, the bibliographies of previously published systematic reviews ${ }^{32}$ were evaluated in an effort to identify any articles not retrieved in our database searches. Only articles in the English language were considered. Original prospective or retrospective studies with at least 5 patients that described operative and clinical outcomes after MIS SI joint fusion using a lateral transarticular approach for SI joint dysfunction were included. Articles that report the use of a dorsal distraction approach, open surgical technique, fusion of the pubic symphysis, single case reports, reports with no clinical data or very limited follow-up, reports on traumatic pelvic ring injuries, ankylosing spondylitis, infection or tumor, or studies that solely evaluated imaging were excluded. In all included studies, patients were diagnosed with SI joint pain using a common approach based on history, physical examination findings and diagnostic SI joint block. All of the included studies reported on patients with degenerative sacroiliitis (i.e., osteoarthritic degeneration of the SI joint) or SI joint disruptions (i.e. joint disruption as a result of isolated SI trauma, pregnancy or other causes).

The following information (where available) was extracted from each study and entered into a spreadsheet: sample size at baseline and each follow-up time point, means and standard deviations for operative parameters (blood loss, length of stay, operating time), and patient reported outcome measures. Pain was reported using either a 0-10 numeric rating scale (NRS), 0-10 visual analog scale (VAS) or a $100 \mathrm{~mm}$ VAS. VAS scores using 0-100 were converted to the $0-10$ scale by dividing all scores by 10 . A number of the studies reported disability as assessed on the Oswestry Disability Index (ODI). ${ }^{33}$ For these studies, means and standard deviations for ODI were included in the spreadsheet. In two cases, ${ }^{34,35}$ study authors were contacted and confirmed that ODI was reported on the $0-50$ scale rather than the $0-100 \%$ scale; for these studies, ODI scores were adjusted prior to analysis. SF-36 and Majeed scoring were reported in two studies. ${ }^{36,37}$ Due to the incongruence in the reported follow-up interval, these measures could not be analyzed quantitatively using meta-analysis.

Change scores (i.e., mean changes from baseline to a particular time point) were reported in only a minority of studies and therefore not summarized. Instead, we report the difference in mean population scores as an approximate substitute. Data quality assurance and control checks were performed to ensure accuracy.

In some cases, studies did not report key values (e.g., standard deviations of population means) or reported values were unexpected; we contacted relevant study authors to obtain the information. In a small number of cases, standard deviation values were unavailable; therefore we imputed these missing values as the mean of available values. Previous publications have failed to show any statistical predictors of change scores, so further extraction of potential covariates from published studies was not performed. ${ }^{38,39}$

In some retrospective case series, cohorts overlapped (e.g. previously reported single center experience included in multi-center case series) or were reported twice (e.g., second article on the same cohort but reported additional findings). Any suspected overlap in studies was verified by contacting the corresponding author. All overlapping cohorts were eliminated, and the data summarized represent unique patients from a total of 12 distinct cohorts; 10 cohorts used a series of triangular porous titanium plasma spray (TPS) coated implants (iFuse Implant System, SI-BONE, Inc., San Jose, CA) and 2 cohorts described the use of a hollow modular anchorage (HMA) screw (Aesculap Ltd., Tuttlingen, Germany) packed with dem- 
ineralized bone matrix.

We summarized data (all of which were continuous variables) using two approaches. First, we calculated mean and $95 \%$ confidence intervals using a random effects meta-analysis (RMA) model. Pain and disability scores were reported in several articles at multiple time points. As the data tended to be mixed (i.e., different studies reported different time points) and because methods for RMA require more complete datasets ${ }^{40}$ a piecewise approach to meta-analysis was performed. That is, individual meta-analytic summaries were performed for each follow-up time point where more than 2 studies were available at that time point. Heterogeneity was assessed using the DerSimonian and Laird (DL) method; ${ }^{41}$ estimates are reported as showing significant heterogeneity if the DL $\mathrm{p}$-value was $<0.05$, moderate heterogeneity if the $\mathrm{p}$ value was between 0.05 and 0.1 and little heterogeneity if the p-value was $>0.1$. Second, we used weighted local regression (LOESS) to fit available data for both SI joint pain and ODI. Weights were the inverse of the variance of each study's mean (i.e., (SD/ $\operatorname{sqrt}(\mathrm{N}))^{-2}$ ). All analysis was done in $\mathrm{R} .{ }^{42}$ Random effects meta-analysis was performed using the metafor package $^{43}$ and LOESS regression was performed using the base stats package. The available literature included two types of devices (triangular implants and HMA screws). Where data were available, device was added as a fixed effect to RMA models.

\section{Results}

Search results yielded 241 records from Pubmed and 297 from EMBASE. Results from each search were downloaded into a .csv file and included title, author, source, ID, and abstract. Each record was reviewed for inclusion using the aforementioned criteria. A total of 538 records were screened; 34 were not in the English language, 372 were not relevant, 2 were duplicates within the same database, 13 were conference abstracts, and 33 identified for further review were not unique between databases. We assessed 84 records for eligibility, 68 of which were excluded for the following reasons: editorial (3), video technique (2), technique description (3), review article (7), biomechanics (6), imaging studies (2), economics (3), anatomy or cadaveric study (7), dorsal distraction technique (5), anterior endoscopic technique (1), sacral fracture (1), pelvic ring fracture or fixation (12), open surgical fusion (6), infection (3), case report (4), device complaints analysis (1), utilization (1), tumor (1) (Figure 1). 16 articles met the inclusion criteria. Two additional articles were identified in a previously published review of MIS SI joint fusion. A total of 18 articles were identified. The study design and number of these are as follows: 10 retrospective single-center case series, ${ }^{12,34,35,37,38,44-48} 2$ prospective single center case series, ${ }^{36,49} 1$ multi-center retrospective case series, ${ }^{50} 1$ single center, ${ }^{27}$ and 2 multicenter $^{26,28}$ comparative cohort studies, one prospective single-arm study, ${ }^{24}$ and one prospective multicenter randomized controlled trial ${ }^{39}$ (Table 1). Two types of implants are represented: 3 studies reported the use of a single hollow modular anchorage (HMA) screw packed with demineralized bone matrix and 15 described the placement of a series (typically 3 ) of triangular, porous titanium plasma spray (TPS) coated implants. After eliminating the overlap in patient cohorts across studies described above, the sum of the literature represents 12 unique studies from 4 different countries with a total of 432 patients; 368 patients from 10 cohorts using triangular TPS coated implants and 64 patients from 2 cohorts using HMA screws (Table 2).

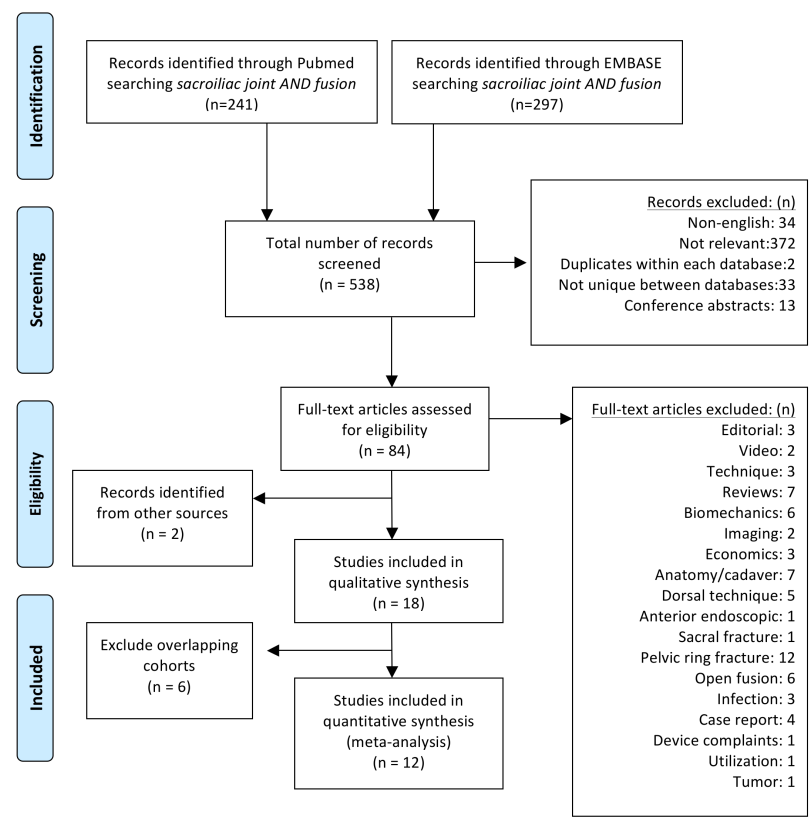

Fig. 1. PRISMA flow diagram detailing search results. 
Table 1. MIS SI joint fusion studies using a lateral transarticular approach.

\begin{tabular}{|c|c|c|c|c|c|c|}
\hline Author, Year & Implant & Study design & $\mathbf{N}$ & $\begin{array}{l}\text { Demographics } \\
\text { Mean ( } \pm \text { SD) or } \\
\text { (range), unless } \\
\text { otherwise specified }\end{array}$ & $\begin{array}{l}\text { Results } \\
\text { Mean }( \pm \text { SD) or (range) } \\
\text { unless otherwise specified } \\
\text { VAS uses a } 0 \text { - } 10 \text { scale unless } \\
\text { otherwise specified }\end{array}$ & Complications (n) \\
\hline Whang, $2015^{39}$ & $\begin{array}{l}\text { Triangular } \\
\text { TPS coated } \\
\text { implants }\end{array}$ & $\begin{array}{l}\text { Prospective, multi-center, } \\
\text { randomized controlled trial } \\
\text { (surgical arm reported herein) }\end{array}$ & 102 & $\begin{array}{l}\text { Age: } 50.2(26-72) \\
\text { years } \\
\text { Sex: } 75 \mathrm{~F} / \mathrm{M} \\
\text { Prior lumbar fusion: } \\
38 \% \\
\text { Follow-up: } 6 \mathrm{mo}\end{array}$ & $\begin{array}{l}\text { VAS: } 8.2(1.2) \text { pre-op, } 2.9 \\
\text { (2.9) at 6mo } \\
\text { ODI: 62.2 (14.5) pre-op, } 31.9 \\
\text { (22.7) at 6mo } \\
\text { SF-36PCS: } 30.2(6.2) \text { pre-op, } \\
\text { 42.8 (10.0) at 6mo } \\
\text { Surgical time: } 44.9(22.3) \\
\text { min } \\
\text { EBL: } 32.7(32.8) \mathrm{mL} \\
\text { Hospital stay: } 0.8 \text { (range } 0-7) \\
\text { days }\end{array}$ & $\begin{array}{l}\text { Trochanteric bursitis (4), surgical wound problems (4), iliac fracture (1), hairline ilium fracture (1), nerve root } \\
\text { impingement (1) }\end{array}$ \\
\hline $\begin{array}{l}\text { Vanaclocha } \\
2014^{49}\end{array}$ & $\begin{array}{l}\text { Triangular } \\
\text { TPS coated } \\
\text { implants }\end{array}$ & Single center case series & 24 & $\begin{array}{l}\text { Age: } 47.4(32-71) \\
\text { years } \\
\text { Sex: } 15 \mathrm{~F} / 9 \mathrm{M} \\
\text { Prior lumbar fusion: } 2 \\
\text { Follow-up: } 23 \mathrm{mo} \\
\text { (1-4.5 years) }\end{array}$ & $\begin{array}{l}\text { VAS: } 8.7 \text { pre-op, } 1.7 \text { at } 1 \mathrm{yr} \text {, } \\
2.1 \text { at } 4.5 \mathrm{yrs} \\
\text { ODI: } 54.1 \text { pre-op, } 14.3 \text { at } 1 \mathrm{yr} \text {, } \\
16.3 \text { at } 4.5 \mathrm{yrs} \\
\text { Surgical time: } 48(40-65) \mathrm{min} \text {, } \\
\text { unilateral cases } \\
\text { EBL: } 58(40-70) \mathrm{mL}\end{array}$ & Immediate post-op pain (4-resolved), temporary post-op radiculopathic pain (2) \\
\hline Rudolf, $2014^{38}$ & $\begin{array}{l}\text { Triangular } \\
\text { TPS coated } \\
\text { implants }\end{array}$ & Single center case series & 17 & $\begin{array}{l}\text { Age: } 58(36-85) \text { years } \\
\text { Sex: } 13 \mathrm{~F} / 4 \mathrm{M} \\
\text { Prior lumbar fusion: } 8 \\
\text { (47\%) } \\
\text { Follow-up: } 60 \mathrm{mo} \\
\text { Bridging bone: } 87 \% \\
(13 / 15)\end{array}$ & $\begin{array}{l}\text { VAS: } 8.3(1.4) \text { pre-op, } 3.4 \\
(2.4) \text { at ly, } 1.4(2.6) \text { at } 2 \mathrm{yrs} \\
2.4(2.2) \text { at } 5 \mathrm{yrs} \\
\text { ODI: } 21.5(22.7) \text { at } 5 \mathrm{yrs} \\
\text { Surgical time: } 65(18) \mathrm{min}\end{array}$ & $\begin{array}{l}\text { No intraoperative complications, hematoma (1), cellulitis (2), deep wound infection secondary to diverticulitis } \\
\text { (1) }\end{array}$ \\
\hline Sachs, $2014^{50}$ & $\begin{array}{l}\text { Triangular } \\
\text { TPS coated } \\
\text { implants }\end{array}$ & Multi-center, Retrospective & 144 & $\begin{array}{l}\text { Age: } 58(30-89) \text { years } \\
\text { Sex: } 30 \mathrm{~F} / 10 \mathrm{M} \\
\text { Prior lumbar fusion: } \\
62 \% \\
\text { Follow-up: } 16 \mathrm{mo} \\
(12-26)\end{array}$ & $\begin{array}{l}\text { VAS: } 8.6 \text { pre-op, } 2.7 \text { at } \\
\text { follow-up } \\
91 \% \text { Very or somewhat } \\
\text { satisfied } \\
91.7 \% \text { would have surgery } \\
\text { again } \\
\text { Surgical time: } 73 \mathrm{~min} \\
\text { EBL: } 31 \mathrm{~mL} \\
\text { Hospital stay: } 0.8 \text { days }\end{array}$ & $\begin{array}{l}\text { No intraoperative complications. } \\
\text { fall (5), trochanteric bursitis (4), piriformis syndrome (3), facet pain (3), contralateral SIJ pain(2), recurrent } \\
\text { pain (3), leg pain (1), toe/foot numbness (2), hematoma (1), low back pain (1), implant revision (1), burning in } \\
\text { upper thigh (1), bladder incontinence (1) }\end{array}$ \\
\hline \multirow{2}{*}{$\begin{array}{l}\text { Ledonio, } \\
2014^{27}\end{array}$} & $\begin{array}{l}\text { Triangular } \\
\text { TPS coated } \\
\text { implants }\end{array}$ & \multirow{2}{*}{$\begin{array}{l}\text { Single center } \\
\text { Retrospective, comparative cohort } \\
\text { study }\end{array}$} & 22 & $\begin{array}{l}\text { MIS Cohort } \\
\text { Age: } 47.9(13.1) \text { years } \\
\text { Sex: } 17 \mathrm{~F} / 5 \mathrm{M} \\
\text { Prior lumbar fusion: } \\
64 \% \\
\text { Follow-up: median } \\
15 \text { mo (12-26) }\end{array}$ & $\begin{array}{l}\text { ODI: } 61.5(12.5) \text { pre-op, } 52 \\
\text { (16.9) at follow-up } \\
\text { Surgical time: } 68.3(26.8) \mathrm{min} \\
\text { EBL: } 40.5(31.4) \mathrm{mL} \\
\text { Hospital Stay: } 2.0(1.5) \text { days }\end{array}$ & $\begin{array}{l}\text { Pulmonary embolism that resolved with treatment (1), revisions due to halo formation on the sacral side with } \\
\text { recurring sacroiliac joint pain ( } 2 \text { ) }\end{array}$ \\
\hline & $\begin{array}{l}4.5 \mathrm{~mm} \\
\text { plate, } \\
\text { autograft }\end{array}$ & & 22 & $\begin{array}{l}\text { Open Cohort } \\
\text { Age: } 51(9.4) \text { years } \\
\text { Sex: } 13 \mathrm{~F} / 9 \mathrm{M} \\
\text { Prior lumbar fusion: } \\
50 \% \\
\text { Follow-up: median } 13 \\
\text { (11-33) mo }\end{array}$ & $\begin{array}{l}\text { ODI: } 61.8(10.8) \text { pre-op, } 47.4 \\
\text { (21.7) at follow-up } \\
\text { Surgical time: } 128(27.9) \mathrm{min} \\
\text { EBL: } 168.8(479.0) \mathrm{mL} \\
\text { Hospital Stay: } 3.3(1.1) \text { days }\end{array}$ & Pulmonary embolism (1), revision due to failed implant and nerve root irritation (2) \\
\hline
\end{tabular}




\begin{tabular}{|c|c|c|c|c|c|c|}
\hline \multirow{2}{*}{ Ledonio $2014^{28}$} & $\begin{array}{l}\text { Triangular } \\
\text { TPS coated } \\
\text { implants }\end{array}$ & \multirow{2}{*}{$\begin{array}{l}\text { Multi-center } \\
\text { Retrospective, comparative cohort } \\
\text { study }\end{array}$} & 17 & $\begin{array}{l}\text { MIS Cohort } \\
\text { Age: median } 66 \\
\text { (39-82) years } \\
\text { Sex: } 11 \mathrm{~F} / 6 \mathrm{M} \\
\text { Prior lumbar fusion: } \\
82 \% \\
\text { Follow-up: } 12 \mathrm{mo}\end{array}$ & 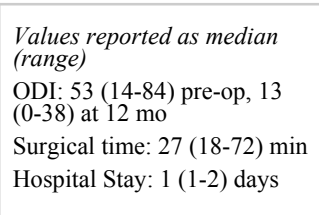 & $\begin{array}{l}\text { Transient trochanteric bursitis (3), hematoma (1), transient toe numbness (1), revision due to malpositioned } \\
\text { implant (1) }\end{array}$ \\
\hline & $\begin{array}{l}4.5 \mathrm{~mm} \\
\text { plate, } \\
\text { autograft }\end{array}$ & & 22 & $\begin{array}{l}\text { Open Cohort } \\
\text { Age: median } 51 \\
\text { (34-74) years } \\
\text { Sex: } 82 \mathrm{~F} / 32 \mathrm{M} \\
\text { Prior lumbar fusion: } \\
47 \% \\
\text { Follow-up: } 24 \mathrm{mo}\end{array}$ & $\begin{array}{l}\text { Values reported as median } \\
\text { (range) } \\
\text { ODI: } 64 \text { (44-78) pre-op, } 46 \\
\text { (10-80) at } 12 \mathrm{mo} \\
\text { Surgical time: } 128 \text { (73-180) } \\
\text { min } \\
\text { Hospital Stay: } 3 \text { (2-6) days }\end{array}$ & Pulmonary embolism (1), revision due to failed implant and nerve root irritation (2) \\
\hline \multirow{2}{*}{$\begin{array}{l}\text { Graham-Smith, } \\
2013^{26}\end{array}$} & $\begin{array}{l}\text { Triangular } \\
\text { TPS coated } \\
\text { implants }\end{array}$ & \multirow{2}{*}{$\begin{array}{l}\text { Multi-center } \\
\text { Retrospective comparative cohort } \\
\text { study }\end{array}$} & 114 & $\begin{array}{l}\text { MIS Cohort } \\
\text { Age: } 57.4(14.0) \text { years } \\
\text { Sex: } 82 \mathrm{~F} / 32 \mathrm{M} \\
\text { Prior lumbar fusion: } \\
47.4 \% \\
\text { Follow-up: } 24 \mathrm{mo}\end{array}$ & $\begin{array}{l}\text { VAS: } 8.3(1.6) \text { pre-op, } 2.3 \\
\text { (2.6) at } 12 \text { mo, } 1.7(2.9) \text { at } \\
24 \mathrm{mo} \\
\text { MCID: } 86 \% \text { reached at } \\
12 \mathrm{mo}, 82 \% \text { at } 24 \mathrm{mo} \\
\text { Surgical time: } 70(24) \mathrm{min} \\
\text { EBL: } 33(27) \mathrm{mL} \\
\text { Hospital stay: } 1.3(0.5) \text { days }\end{array}$ & $\begin{array}{l}\text { No intraoperative complications. } \\
\text { Postop repositioning of implants (4), 3.5\% (4/114), cellulitis (3), facet pain (4) piriformis syndrome (2), } \\
\text { trochanteric bursitis (2), wound infection (1) }\end{array}$ \\
\hline & $\begin{array}{l}\text { Screws, } \\
\text { plates }\end{array}$ & & 149 & $\begin{array}{l}\text { Open Cohort } \\
\text { Age: } 45.8(11.3) \text { years } \\
\text { Sex: } 103 \mathrm{~F} / 46 \mathrm{M} \\
\text { Prior lumbar fusion: } \\
23.5 \% \\
\text { Follow-up: } 24 \mathrm{mo}\end{array}$ & $\begin{array}{l}\text { VAS: } 7.1(1.9) \text { pre-op, } 4.6 \\
\text { (3.0) at } 12 \text { mo, 5.6 (2.9) at } \\
24 \mathrm{mo} \\
\text { MCID: } 61 \% \text { reached at } \\
12 \mathrm{mo}, 50 \% \text { at } 24 \mathrm{mo} \\
\text { Surgical time: } 163(25) \mathrm{min} \\
\text { EBL: } 288(182) \mathrm{mL} \\
\text { Hospital stay: } 5.1(1.9) \text { days }\end{array}$ & $\begin{array}{l}\text { No intraoperative. } \\
\text { Postop removal of implants ( } 66), 44 \%(66 / 149) \text {. Bone fragment (1), cellulitis (1), leg pain (3), postoperative } \\
\text { neuropathy (4), pulmonary embolism (2), trochanteric bursitis (4), wound infection (3) }\end{array}$ \\
\hline Duhon, $2013^{24}$ & $\begin{array}{l}\text { Triangular } \\
\text { TPS coated } \\
\text { implants }\end{array}$ & $\begin{array}{l}\text { Multi-center, Prospective, efficacy } \\
\text { (32) and safety (94) cohorts }\end{array}$ & 32,94 & $\begin{array}{l}\text { Age: } 50.2(12.6) \text { years } \\
\text { Sex: } 21 \mathrm{~F} / 11 \mathrm{M} \\
\text { Prior lumbar fusion: } \\
69 \% \\
\text { Follow-up: } 6 \mathrm{mo}\end{array}$ & $\begin{array}{l}\text { VAS }(0-100 \mathrm{~mm}): 76.2(16.2) \\
\text { pre-op, } 29.3(23.3) \text { at } 6 \mathrm{mo} \\
\text { ODI: } 55.3(10.7) \text { pre-op, } 38.9 \\
(18.5) \text { at } 6 \mathrm{mo} \\
\text { SF-36 PCS: } 30.7 \text { (4.3) pre-op, } \\
37(10.7) \text { at } 6 \mathrm{mo} \\
88.5 \%(23 / 26) \text { success rate } \\
\text { Surgical time: } 48(16.1) \mathrm{min} \\
\text { EBL: } 59 \text { (95) mL } \\
\text { Hospital stay: } 0.8 \text { days }\end{array}$ & $\begin{array}{l}\text { No implant revision or removal, } 6 \text { AEs probably or definitely procedure-related ( } 2 \text { nausea, } 2 \text { wound infections, } \\
1 \text { cellulitis, } 1 \text { buttock pain) }\end{array}$ \\
\hline Sachs, $2013^{44}$ & $\begin{array}{l}\text { Triangular } \\
\text { TPS coated } \\
\text { implants }\end{array}$ & $\begin{array}{l}\text { Single center, } \\
\text { Retrospective case series }\end{array}$ & 40 & $\begin{array}{l}\text { Age: } 58(30-81) \text { years } \\
\text { Sex: } 30 \mathrm{~F} / 10 \mathrm{M} \\
\text { Prior lumbar fusion: } \\
30 \% \\
\text { Follow-up: } 12 \mathrm{mo}\end{array}$ & $\begin{array}{l}\text { VAS: } 8.7(1.5) \text { pre-op, } 0.9 \\
\text { (1.6) at } 12 \mathrm{mo} \\
98 \% \text { reached MCID } \\
100 \% \text { patient satisfaction }\end{array}$ & Piriformis syndrome (1), new LBP (1), facet joint pain (8), trochanteric bursitis (2) \\
\hline $\begin{array}{l}\text { Cummings, } \\
2013^{45}\end{array}$ & $\begin{array}{l}\text { Triangular } \\
\text { TPS coated } \\
\text { implants }\end{array}$ & $\begin{array}{l}\text { Single center, Retrospective case } \\
\text { series }\end{array}$ & 18 & $\begin{array}{l}\text { Age: } 64(39-81) \text { years } \\
\text { Sex: } 12 \mathrm{~F} / 6 \mathrm{M} \\
\text { Prior lumbar fusion: } \\
61 \% \\
\text { Follow-up: } 12 \mathrm{mo}\end{array}$ & $\begin{array}{l}\text { VAS: } 8.9(1.9) \text { pre-op, } 2.3 \\
\text { (2.1) at } 12 \mathrm{mo} \\
90 \% \text { reached MCID } \\
\text { ODI: } 52.6(18.8) \text { pre-op, } 13.2 \\
\text { (12.6) at } 12 \mathrm{mo} \\
\text { SF-12 PCS: } 37.8(10.4) \\
\text { pre-op, } 44.6(10.5) \text { at } 12 \mathrm{mo}\end{array}$ & Trochanteric bursitis (3), hematoma (1), fluid retention (1), toe numbness (1), implant malposition (1) \\
\hline
\end{tabular}




\begin{tabular}{|c|c|c|c|c|c|c|}
\hline Gaetani, $2013^{35}$ & $\begin{array}{l}\text { Triangular } \\
\text { TPS coated } \\
\text { implants }\end{array}$ & $\begin{array}{l}\text { Single center, } \\
\text { Retrospective case series }\end{array}$ & 12 & $\begin{array}{l}\text { Age: } 53.2(36-71) \\
\text { years } \\
\text { Sex: } 12 \mathrm{~F} \\
\text { Prior lumbar fusion: } \\
8.3 \% \\
\text { Follow-up: } 10 \mathrm{mo} \\
(8-18)\end{array}$ & $\begin{array}{l}\text { VAS: } 7.7(1.3) \text { pre-op, } 3(1.2) \\
\text { at follow-up } \\
\text { ODI: } 31.4(6.3) \text { pre-op, } 12 \\
\text { (3.5) at follow-up } \\
\text { RDQ: } 17.6(1 \text { pre-op, } 3(4.1) \\
\text { at follow-up } \\
\text { Surgical time: } 65(16) \mathrm{min} \\
\text { EBL: }<45 \mathrm{~mL} \\
3 \text { month CT scans show } \\
\text { initial fusion }\end{array}$ & Local hematoma (2), low back pain (1) \\
\hline $\begin{array}{l}\text { Schroeder, } \\
2013^{34}\end{array}$ & $\begin{array}{l}\text { Triangular } \\
\text { TPS coated } \\
\text { implants }\end{array}$ & $\begin{array}{l}\text { Single center, } \\
\text { Retrospective case series }\end{array}$ & 6 & $\begin{array}{l}\text { Age: } 50(25-60) \text { years } \\
\text { Sex: } 6 \mathrm{~F} / 0 \mathrm{M} \\
\text { Prior lumbar fusion: } \\
100 \% \text { (deformity } \\
\text { correction) } \\
\text { Follow-up: } 10.25 \mathrm{mo} \\
\text { (4-15) }\end{array}$ & $\begin{array}{l}\text { VAS: } 7.83 \text { pre-op, } 2.67 \text { at } \\
\text { follow-up } \\
\text { ODI: } 22.1 \text { pre-op, } 10.5 \text { at } \\
\text { follow-up } \\
\text { Hospital stay: } 2 \text { days (range } \\
1-4 \text { ) } \\
\text { Bony bridging seen in } 4 \\
\text { patients }\end{array}$ & No intraoperative or post-operative complications. \\
\hline \multirow{3}{*}{ Rudolf, $2013^{46}$} & \multirow{3}{*}{$\begin{array}{l}\text { Triangular } \\
\text { TPS coated } \\
\text { implants }\end{array}$} & \multirow{3}{*}{ Single center, Sub-group analysis } & 18 & $\begin{array}{l}\text { *No prior fusion } \\
\text { Age: } 49(12) \\
\text { Sex: } 12 \mathrm{~F} / 6 \mathrm{M}\end{array}$ & $\begin{array}{l}\mathrm{n} \text { Rudolf } 2012 \text { to assess effect of } \\
\text { VAS decrease at } 12 \mathrm{mo}:-5.94 \\
(3.3) \\
\text { VAS decrease at } 24 \mathrm{mo}:-5.47 \\
(2.88) \\
\text { Surgical time: } 60(19) \mathrm{min}\end{array}$ & Superficial cellulitis (2), wound infection (1), revision for implant malposition (1) \\
\hline & & & 15 & $\begin{array}{l}\text { *Prior lumbar spinal } \\
\text { fusion } \\
\text { Age: } 58(11) \\
\text { Sex: } 11 \mathrm{~F} / 4 \mathrm{M}\end{array}$ & $\begin{array}{l}\text { VAS decrease at } 12 \mathrm{mo}:-3.5 \\
(3.46) \\
\text { VAS decrease at } 24 \mathrm{mo}:-5.81 \\
(3.5) \\
\text { Surgical time: } 64(19) \mathrm{min}\end{array}$ & Superficial cellulitis (2), buttock hematoma (1), revision for implant malposition (1) \\
\hline & & & 7 & $\begin{array}{l}\text { *Concomitant lumbar } \\
\text { pathology treated } \\
\text { non-surgically } \\
\text { Age: } 58(17) \text { Sex: } 3 \mathrm{~F} / \\
\text { 4M }\end{array}$ & $\begin{array}{l}\text { VAS decrease at } 12 \mathrm{mo}:-3.71 \\
(3.11) \\
\text { VAS decrease at } 24 \mathrm{mo}:-4.79 \\
(4.28) \\
\text { Surgical time: } 64(19) \mathrm{min}\end{array}$ & None reported \\
\hline Rudolf, $2012^{47}$ & $\begin{array}{l}\text { Triangular } \\
\text { TPS coated } \\
\text { implants }\end{array}$ & $\begin{array}{l}\text { Single center, } \\
\text { Retrospective case series }\end{array}$ & 50 & $\begin{array}{l}\text { Age: } 54(24-85) \text { years } \\
\text { Sex: } 34 \mathrm{~F} / 16 \mathrm{M} \\
\text { Prior lumbar fusion: } \\
44 \% \\
\text { Follow-up: } 40 \mathrm{mo} \\
(24-56)\end{array}$ & $\begin{array}{l}\text { VAS: } 7.6 \text { pre-op, } 2.0 \text { at } \\
\text { follow-up } \\
82 \% \text { reached MCID } \\
82 \% \text { patient satisfaction } \\
\text { Surgical time: } 65(26) \text { min }\end{array}$ & $\begin{array}{l}\text { superficial cellulitis (3), } \\
\text { deep wound infection (1), } \\
\text { hematoma (2), reoperation (3) }\end{array}$ \\
\hline Sachs, $2012^{48}$ & $\begin{array}{l}\text { Triangular } \\
\text { TPS coated } \\
\text { implants }\end{array}$ & $\begin{array}{l}\text { Single center, Retrospective case } \\
\text { series }\end{array}$ & 11 & $\begin{array}{l}\text { Age: } 65(45-82) \text { years } \\
\text { Sex: } 10 \mathrm{~F} / 1 \mathrm{M} \\
\text { Prior lumbar fusion: } \\
18 \% \\
\text { Follow-up: } 12 \mathrm{mo}\end{array}$ & $\begin{array}{l}\text { VAS: } 7.9(2.2) \text { pre-op, } 2.3 \\
\text { (3.1) at } 12 \mathrm{mo} \\
\text { Surgical time: } 77.5(31.8) \\
\text { min } \\
\text { EBL: } 21.8(18.9) \mathrm{mL}\end{array}$ & Piriformis syndrome (1), low back pain (1) \\
\hline Mason, $2013^{36}$ & $\begin{array}{l}\text { HMA screw } \\
\text { packed with } \\
\text { DBM }\end{array}$ & Prospective case series & 55 & $\begin{array}{l}\text { Age: } 57 \text { years } \\
\text { Sex: } 46 \mathrm{~F} / 9 \mathrm{M} \\
\text { Prior lumbar fusion: } \\
40 \% \\
\text { Follow-up: } 36 \text { (12-84) } \\
\text { mo }\end{array}$ & $\begin{array}{l}\text { VAS: } 8.05 \text { (1.9) pre-op, } 4.48 \\
\text { (2.81) at follow-up } \\
\text { SF-36PCS: } 26.6(15.2) \\
\text { pre-op, } 43 \text { (22.68) follow-up } \\
\text { Majed: } 36.18(15.08) \text { pre-op, } \\
\text { 64.78 (20.18) follow-up }\end{array}$ & Post-op nerve pain requiring reoperation (2) \\
\hline
\end{tabular}




\begin{tabular}{|c|c|c|c|c|c|c|}
\hline $\begin{array}{l}\text { Khurana, } \\
2009^{77}\end{array}$ & $\begin{array}{l}\text { HMA screw } \\
\text { packed with } \\
\text { DBM }\end{array}$ & Retrospective case series & 15 & $\begin{array}{l}\text { Age: } 48.7 \text { (37.3-62.6) } \\
\text { years } \\
\text { Sex: } 11 \mathrm{~F} / 4 \mathrm{M} \\
\text { Prior lumbar fusion: } \\
40 \% \\
\text { Follow-up: } 17 \text { (9-39) } \\
\text { mo }\end{array}$ & $\begin{array}{l}\text { SF-36PCS: } 28.49(11.24) \\
\text { pre-op, 51.38 (9.87) at } \\
\text { follow-up } \\
\text { Majeed: } 37(18-54) \text { pre-op, } \\
79(63-96) \text { at follow-up } \\
\text { Good to excellent results: } 13 / \\
15 \\
\text { EBL: }<50 \mathrm{ml} \\
\text { Hospital stay: } 2.7 \text { (1-7) days }\end{array}$ & No post-operative neurological or wound complications. \\
\hline $\begin{array}{l}\text { Al-Khayer, } \\
2008^{12}\end{array}$ & $\begin{array}{l}\text { HMA screw } \\
\text { packed with } \\
\text { DBM }\end{array}$ & Retrospective case series & 9 & $\begin{array}{l}\text { Age: } 42(35-56) \text { years } \\
\text { Sex: } 9 F \\
\text { Follow-up: } 40 \text { (24-70) } \\
\text { mo }\end{array}$ & $\begin{array}{l}\text { VAS: } 8.1(7-9) \text { pre-op, } 4.6 \\
\text { (3-7) follow-up } \\
\text { ODI: } 59(34-70) \text { to } 45 \\
\text { (28-60) } \\
\text { EBL: }<50 \mathrm{ml} \\
\text { Hospital stay: } 6.9(2-11) \text { days } \\
\text { Return to work: } 44.44 \%\end{array}$ & Deep wound infection requiring debridement and IV antibiotics (1) \\
\hline
\end{tabular}

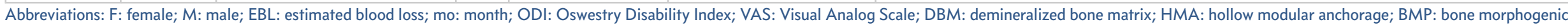
protein. 
Reported operative parameters were procedure time, estimated blood loss (EBL), and hospital length of stay (LOS). Mean procedure time, reported in 11 studies, ranged from 27 to 78 minutes. The random effects meta-analysis (RMA) mean (95\% CI) procedure time was 59 minutes (50.9-66.9) (range 27-78 min) with significant heterogeneity across studies. Mean EBL was reported in 6 studies and ranged from 31 to $59 \mathrm{cc}$. In 5 additional studies, EBL was reported as $<50$ or $<45 \mathrm{cc}$; these were converted to $50 \mathrm{cc}$ for analysis. The RMA mean EBL was $36.9 \mathrm{cc}$ (31.4 - 42.38) with moderate heterogeneity across studies. Mean hospital LOS, reported in 9 studies, ranged from $0.78-6.9$ days (range $0-7$ days). The RMA mean LOS was 1.7 days (1.2 - 2.2) with significant heterogeneity across studies.

Pain severity was reported in all studies. The number

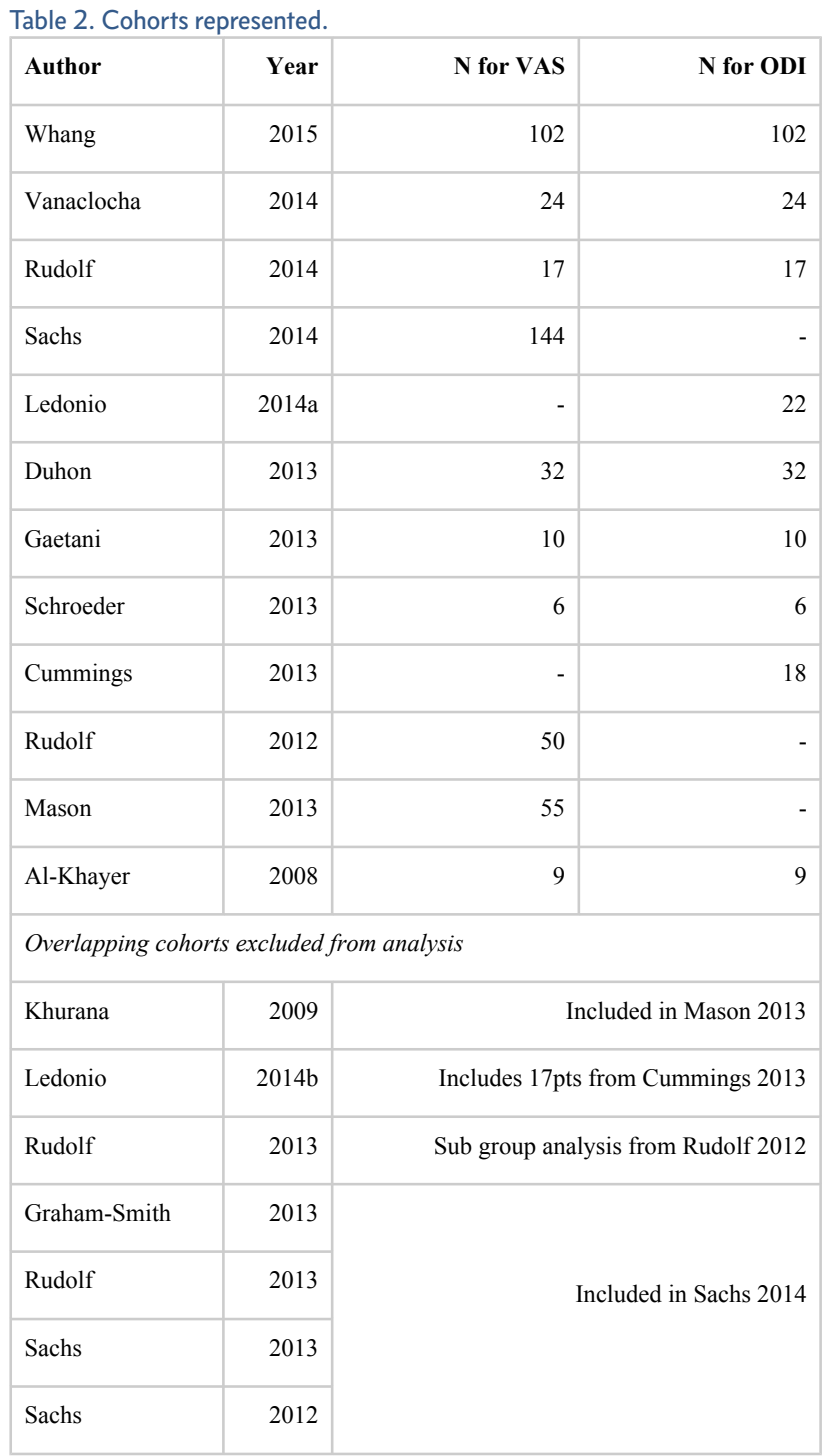

Downloaded from http://ijssurgery.com/ by guest on April 25, 2023 of unique cohorts reporting values by time were: baseline ( 9 studies; 7 triangular implant, 2 HMA screw), 6 months (4 studies, all triangular implant), 12 months (5 studies, all triangular implant), 24 months (2 studies, all triangular implant), 36 months (3 studies; 1 triangular implant, 2 HMA screw), 48 months ( 1 study, triangular implant) and 60 months (1 study, triangular implant) (Figure 2). RMA was performed only to summarize values at baseline, 6 , 12, 24 and 36 months. The baseline RMA mean pain score was 8.1 (7.8-8.4). The 6-month score was 2.8 (2.4-3.2), representing an approximate 5.2-point drop. The 12-month score was 2.7 (2.1-3.3), representing an approximate 5.3-point drop. The 24-month score of 2.0 (1.4- 2.5) represented the triangular implant only. The 36-month score was 3.7 (2.0-5.4); 83\% of which represent outcomes with HMA screws. Outcomes at this timepoint were significantly different $(\mathrm{p}<.0001)$ by implant type: mean (SD) score was 2.0 (1.9) for the triangular implant and 4.6 (2.5) for the HMA screw. Significant heterogeneity was observed for the baseline, 12- and 36-month scores but not the 6- or 24-month scores.

ODI was used in several studies, with values reported at baseline (8 studies), 6 months (4 studies,), 12 months (5 studies), 24 months ( 1 study), 36 months (2 studies), 48 and 60 months (1 study each time point) (Figure 3). Outcomes for one HMA screw cohort were available at baseline and 36 months only. ODI decreased from an RMA mean $(95 \% \mathrm{CI})$ of 56.6 (51.8-61.3) at baseline to 30.3 (22.5-38.0) at 6 months and 25.1 (12.3-37.9) at 12 months (Figure 2). Significant $(\mathrm{p}<.05)$ heterogeneity was observed for the baseline, 6 - and 12 -month values. The mean changes

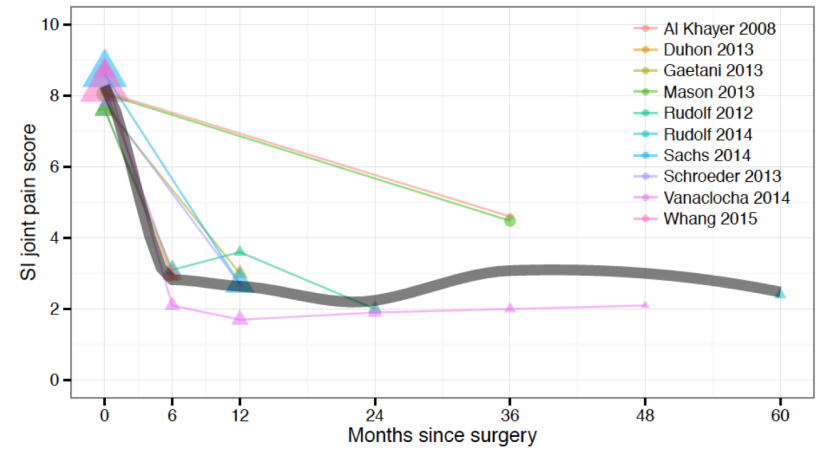

Fig. 2. SI joint pain score by months since surgery across studies. Time $0=$ baseline (pre-surgery). Dot area is proportional to the inverse variance of each study's estimate. The gray line shows an inverse-variance weighted LOESS regression fit. 
from baseline to 6 months and 12 months were approximately 25 and 30 points, respectively. The 36-month RMA mean (95\% CI) of 30.4 (2.0-58.8) was calculated from 9 patients with the HMA screw and 13 patients with the triangular implant. However, the mean outcomes reported from each cohort were significantly different. Although both groups were similar in terms of baseline disability, the triangular implant cohort showed an improvement of 2.7 times that of the HMA screw cohort (baseline: 59, 54.1; 36-month: 45, 16 for the HMA screw and triangular implant, respectively).

Patient quality of life measured on the SF-36 physical component score (PCS) was not consistently reported across studies, therefore RMA methods were not utilized. Scores were available at baseline and 6-months from 2 prospective multi-center trials for the triangular implant cohort and at 36-months for one HMA screw cohort. Improvements were consistent between the 2 studies of triangular implants; $;^{24,39}$ 30.2 (6.2) and 30.7 (4.3) at baseline to 42.8 (10.0) and 37 (10.7) at 6 months, respectively. The HMA screw cohort showed a similar improvement from
$26.6(15.2)$ at baseline to $43(22.68)$ at 36 -months. ${ }^{36}$

Majeed score, ${ }^{51}$ an assessment tool used for grading the outcome after pelvic fractures, was reported in one HMA screw cohort; mean (SD) score improved from 36.18 (15.08) at baseline to $64.78(20.18)$ at 36-months.

Due to variability across studies in adverse event reporting, meta-analytic methods were not used. Instead, rates were calculated as the number of events divided by the total number of patients. In the 12 cohorts represented (432 patients treated), the most common events were surgical wound problems (17 events, 3.9\% rate), trochanteric bursitis (8 events, $2.2 \%$ ), facet pain (3 events, $0.8 \%$ ), recurrent SI pain (i.e., initial improvement in SI joint pain followed by pain recurrence, 3 events, $0.8 \%$ ), and toe/foot numbness ( 2 events, $0.5 \%$ ) (Table 3 ). Nerve root impingement requiring revision occurred in 9 subjects $(2.1 \%)$. There were no deaths.

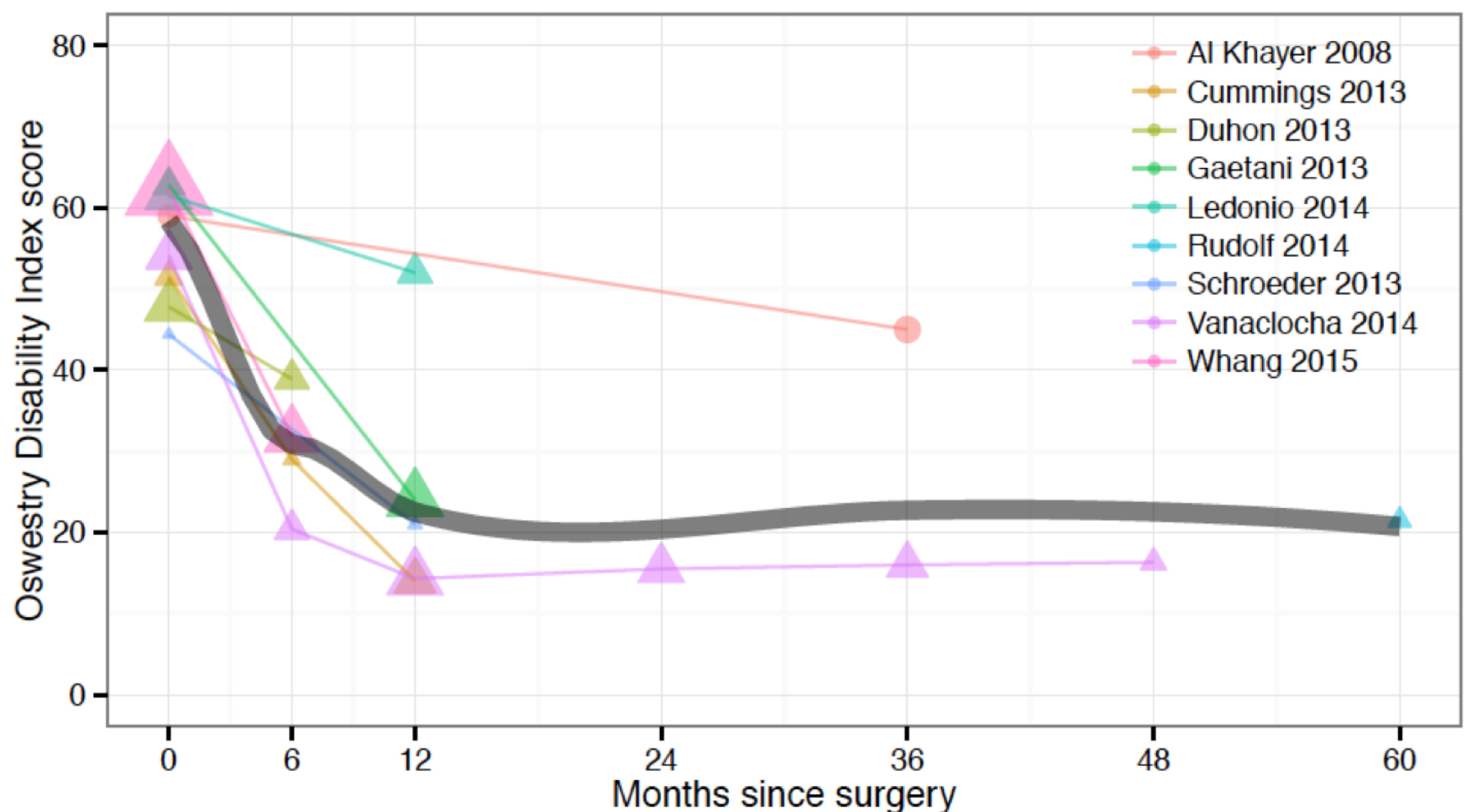

Fig. 3. Oswestry Disability Index by months since surgery across studies. Time 0 = baseline (pre-surgery). Dot area is proportional to the inverse variance of each study's estimate. The gray line shows an inverse-variance weighted LOESS regression fit. 


\section{Discussion}

Minimally invasive fusion of the SI joint has been performed with several types of implants using either a dorsal joint distraction technique (titanium cages packed with bone morphogenic protein ${ }^{52}$, allograft bone dowels ${ }^{53}$, autograft iliac bone plugs ${ }^{54}$ ) or, more commonly, through a lateral transarticular approach using either hollow modular anchorage screws packed with demineralized bone matrix ${ }^{12,36,37}$ or triangular titanium plasma spray (TPS) coated implants..$^{24,26-28,34,35,38,39,44-50}$ As the two techniques are fundamentally different and body of literature supporting the latter approach is comparatively more substantial, the current systematic review focused on the lateral transarticular approach only. Compared to open SI joint fusion, MIS SI joint fusion as reported herein is associated with less blood loss, a shorter hospital stay and improved clinical responses. ${ }^{26-28}$

\begin{tabular}{|c|c|}
\hline Complication & $\mathrm{n}$ \\
\hline $\begin{array}{l}\text { Surgical wound problems (including hematoma, wound infec- } \\
\text { tion, cellulitis) }\end{array}$ & 17 \\
\hline Iliac fracture & 1 \\
\hline Hairline ilium fracture at caudal implant & 1 \\
\hline Pulmonary embolism & 1 \\
\hline Nerve root impingement requiring revision & 9 \\
\hline Transient post-op radiculopathic pain & 3 \\
\hline Buttock pain & 1 \\
\hline Low back pain & 2 \\
\hline Trochanteric bursitis & 8 \\
\hline Piriformis syndrome & 3 \\
\hline Facet pain & 3 \\
\hline Contralateral SIJ pain & 2 \\
\hline Recurrent pain & 3 \\
\hline Leg pain & 1 \\
\hline Toe/foot numbness & 2 \\
\hline Bladder incontinence & 1 \\
\hline Total & $\begin{array}{l}57 / 432 \\
(7.6 \%)\end{array}$ \\
\hline Revision rate & $\begin{array}{r}9 / 432 \\
(2.1 \%)\end{array}$ \\
\hline
\end{tabular}

Not surprisingly, the use of minimally invasive fusion for SI joint pathology has overtaken open approaches. $^{55}$

Our analysis of intraoperative outcomes showed a mean procedure time of approximately one hour, mean estimated blood loss of $50 \mathrm{cc}$ and a mean length of stay of approximately 1.7 days. These procedure-related variables compare favorably to open surgical SI joint fusion cohorts, which reported mean procedure times of 128-163 minutes, EBL of 288-682 cc, and mean hospital LOS of 3.3-5.2 days. $^{26,28}$

All studies reported a rapid reduction in SI joint pain after surgery; compared to pre-surgical values, mean pain scores were reduced at all assessed time points by approximately 5 points on the $0-10$ scale, representing a substantial clinical benefit on average (Figure 2). In parallel with pain scores, disability as measured by Oswestry Disability Index also showed marked improvements after surgery with mean changes at 6 months of approximately 25 points and mean changes at 12 months and thereafter of approximately 30 points (Figure 3 ). These mean changes exceed the threshold of substantial clinical benefit (18.8 point change from baseline) ${ }^{56}$ In comparison to the modest changes in pain and disability in similar patients treated with non-surgical management ${ }^{39}$, improvements after minimally invasive SI joint fusion using a lateral transarticular approach appear to be substantial and clinically important. It is encouraging to see that studies with longer term outcomes consistently showed sustained positive outcomes on VAS and ODI over time. ${ }^{38,49}$

We did not use meta-analysis to summarize adverse event rates across published studies, as methods used to collect such events were not specified. It is important to note that serious adverse events related to the device and placement procedure appeared to be uncommon..$^{57}$ Two ongoing prospective clinical trials included in this systematic review ${ }^{24,39}$ collect all negative changes in health as adverse events according to an international clinical trial standard (ISO 14155:2011) definition. Complications reported to date from these trials are similar in nature to those summarized herein. 
Of 18 studies included in this systematic review, 83\% described the use of a series of triangular TPS coated implants. This uniquely designed implant system works on the well-accepted principle of joint stabilization followed by long-term fusion. The porous coating of the device is similar to that used in total joint replacement surgery. These types of coatings are designed to promote biological fixation of the bone to the implant and a biomechanically sound construct. The immediate solid fixation is akin to that afforded in press fit total joints. Fusion of the SI joint using these implants has been demonstrated in two long-term studies. ${ }^{38,49}$

SI joint dysfunction is associated with a significant burden of disease ${ }^{58}$ similar to that observed with other prominent orthopedic conditions, such as hip and knee osteoarthritis, spinal stenosis and degenerative spondylolisthesis, all of which are treated surgically. Therefore, it is reasonable to consider surgical treatment for cases of sacroiliac pain that do not respond to non-operative therapy. The meta-analysis performed in this systematic review demonstrated that patients who fail conservative therapy and have a positive diagnostic SI joint block may clinically benefit from minimally invasive sacroiliac joint fusion using a lateral transarticular approach.

There are several strengths and weaknesses to this study. Strengths include similarity of diagnosis, surgical approach (lateral, transarticular) and follow-up assessments (VAS, ODI) across studies. Significant heterogeneity was observed in many estimates; there were distinct differences in both operative measures and patient reported outcomes between the 2 implant types. Although overall outcomes after MIS SI joint fusion are favorable, the results described herein may not be transferable to other types of implants or approaches to achieving arthrodesis of the SI joint. The HMA screws are not currently FDA-cleared for MIS SI joint fusion and are not commercially available in the US.

A major limitation of this systematic review is the dearth of high quality evidence for MIS SI joint fusion; only one level I randomized trial and 3 prospective studies were available. We identified overlap in published cohorts that reduced the total number of available subjects. Substantial variation across studies in both the follow-up interval and the types of outcome measures employed prohibited the use of meta-analytic methods for certain variables as well as for long-term outcomes. Outcomes between implant types varied, in some instances substantially (ODI and VAS). The PRISMA standard suggests a review protocol, which was not done for this assessment. Individual studies could have been biased due to selfreport, regression to the mean, or other factors; these potential biases could not be addressed by our study. However, we note that improvement in pain, disability and quality of life in the non-surgical control group of the one prospective randomized trial was minimal. ${ }^{39}$ In addition, we were unable to assess potential bias across studies due to publication bias or selective within-study reporting. We note that prospective trials required adverse event reporting according to an international clinical trial standard; this approach is typically not used in retrospective case series. Finally, some of the studies published on the triangular implants were sponsored by the manufacturer; however, most spine surgery device studies are industry-sponsored. ${ }^{59}$

\section{Conclusions}

A systematic review and meta-analysis of published studies of MIS SI joint fusion using a lateral transarticular approach in patients with symptoms due to SI joint dysfunction due to degenerative sacroiliitis or SI joint disruptions recalcitrant to non-surgical treatment show consistent and clinically important improvements in pain and associated disability. Furthermore, the minimally invasive characteristics of this procedure were confirmed as evidenced by minimal blood loss, a short operative time and brief length of stay.

\section{References}

1. Salomon JA, Vos T, Hogan DR, et al. Common values in assessing health outcomes from disease and injury: disability weights measurement study for the Global Burden of Disease Study 2010. Lancet. 2012;380(9859):2129-2143. doi:10.1016/ S0140-6736(12)61680-8.

Downloaded from http://ijssurgery.com/ by guest on April 25, 2023 
2. Weksler N, Velan GJ, Semionov M, et al. The role of sacroiliac joint dysfunction in the genesis of low back pain: the obvious is not always right. Arch Orthop Trauma Surg. 2007;127(10):885-888. doi:10.1007/s00402-007-0420-x.

3. Katz JN. Lumbar disc disorders and low-back pain: socioeconomic factors and consequences. $J$ Bone Joint Surg Am. 2006;88 Suppl 2:21-24. doi:10.2106/JBJS.E.01273.

4. Kitayuguchi J, Kamada M, Okada S, Kamioka H, Mutoh Y. Association between musculoskeletal pain and trips or falls in rural Japanese communitydwelling older adults: A cross-sectional study. Geriatr Gerontol Int. January 2014. doi:10.1111/ggi.12228. 5. Sturesson B, Selvik G, Udén A. Movements of the sacroiliac joints. A roentgen stereophotogrammetric analysis. Spine. 1989;14(2):162-165.

6. Fortin JD, Dwyer AP, West S, Pier J. Sacroiliac joint: pain referral maps upon applying a new injection/arthrography technique. Part I: Asymptomatic volunteers. Spine. 1994;19(13):1475-1482.

7. Dreyfuss P, Henning T, Malladi N, Goldstein B, Bogduk N. The Ability of Multi-Site, Multi-Depth Sacral Lateral Branch Blocks to Anesthetize the Sacroiliac Joint Complex. Pain Med.

2009;10(4):679-688. doi:10.1111/

j.1526-4637.2009.00631.x.

8. Schwarzer AC, Aprill CN, Bogduk N. The sacroiliac joint in chronic low back pain. Spine. 1995;20(1):31-37.

9. Sembrano JN, Polly DW. How often is low back pain not coming from the back? Spine.

2009;34(1):E27-E32. doi:10.1097/

BRS.0b013e31818b8882.

10. Bernard TN, Kirkaldy-Willis WH. Recognizing specific characteristics of nonspecific low back pain. Clin Orthop. 1987;(217):266-280.

11. Maigne JY, Aivaliklis A, Pfefer F. Results of sacroiliac joint double block and value of sacroiliac pain provocation tests in 54 patients with low back pain. Spine. 1996;21(16):1889-1892.

12. Al-Khayer A, Hegarty J, Hahn D, Grevitt MP. Percutaneous sacroiliac joint arthrodesis: a novel technique. J Spinal Disord Tech. 2008;21(5):359-363. doi:10.1097/BSD.0b013e318145ab96.

13. Slinkard N, Agel J, Swiontkowski MF. Documentation of outcomes for sacroiliac joint fusion: does prior spinal fusion influence the outcome? Eur Spine J Off Publ Eur Spine Soc Eur Spinal Deform Soc Eur Sect Cerv Spine Res Soc. 2013;22(10):2318-2324. doi:10.1007/s00586-013-2968-1.

14. Slipman CW, Shin CH, Patel RK, et al. Etiologies of failed back surgery syndrome. Pain Med Malden Mass. 2002;3(3):200-214; discussion 214-217. doi:10.1046/j.1526-4637.2002.02033.x.

15. Bolt PM, Wahl MM, Schofferman J. The Roles of the Hip, Spine, Sacroiliac Joint, and Other Structures in Patients with Persistent Pain after Back Surgery. Semin Spine Surg. 2008;20(1):14-19. doi:10.1053/j.semss.2007.11.003.

16. Yoshihara H. Sacroiliac joint pain after lumbar/ lumbosacral fusion: current knowledge. Eur Spine $J$ Off Publ Eur Spine Soc Eur Spinal Deform Soc Eur Sect Cerv Spine Res Soc. 2012;21(9):1788-1796. doi:10.1007/s00586-012-2350-8.

17. Maigne JY, Planchon CA. Sacroiliac joint pain after lumbar fusion. A study with anesthetic blocks. Eur Spine J Off Publ Eur Spine Soc Eur Spinal Deform Soc Eur Sect Cerv Spine Res Soc. 2005;14(7):654-658. doi:10.1007/s00586-004-0692-6.

18. Ha K-Y, Lee J-S, Kim K-W. Degeneration of sacroiliac joint after instrumented lumbar or lumbosacral fusion: a prospective cohort study over fiveyear follow-up. Spine. 2008;33(11):1192-1198. doi:10.1097/BRS.0b013e318170fd35.

19. DePalma MJ, Ketchum JM, Saullo TR. Etiology of Chronic Low Back Pain in Patients Having Undergone Lumbar Fusion. Pain Med. 2011;12(5):732-739. doi:10.1111/j.1526-4637.2011.01098.x.

20. Spiker WR, Lawrence BD, Raich AL, Skelly AC, Brodke DS. Surgical versus injection treatment for injection-confirmed chronic sacroiliac joint pain. Evid-Based Spine-Care J. 2012;3(4):41-53.

21. Lorio MP, Rashbaum R. ISASS Policy Statement - Minimally Invasive Sacroiliac Joint Fusion. Int J Spine Surg. 2014;8:Article 25.

22. Patel N. Twelve-Month Follow-Up of a Randomized Trial Assessing Cooled Radiofrequency Denervation as a Treatment for Sacroiliac Region Pain. Pain Pract Off J World Inst Pain. January 2015. doi:10.1111/papr.12269.

23. Goldthwait JE, Osgood RB. A consideration of the pelvic articulations from an anatomical, pathological and clinical standpoint. Boston Med Surg J. 
1905;152(21):593-601.

24. Duhon B, Cher D, Wine K, Lockstadt H, Kovalsky D, Soo C-L. Safety and 6-month effectiveness of minimally invasive sacroiliac joint fusion: a prospective study. Med Devices Evid Res. 2013;6:219-229. doi:10.2147/MDER.S55197.

25. Waisbrod H, Krainick JU, Gerbershagen HU. Sacroiliac joint arthrodesis for chronic lower back pain. Arch Orthop Trauma Surg Arch Für Orthop UnfChir. 1987;106(4):238-240.

26. Graham Smith A, Capobianco R, Cher D, et al. Open versus minimally invasive sacroiliac joint fusion: a multi-center comparison of perioperative measures and clinical outcomes. Ann Surg Innov Res. 2013;7(1):14. doi:10.1186/1750-1164-7-14.

27. Ledonio CGT, Polly DW, Swiontkowski MF. Minimally invasive versus open sacroiliac joint fusion: are they similarly safe and effective? Clin Orthop. 2014;472(6):1831-1838. doi:10.1007/ s11999-014-3499-8.

28. Ledonio C, Polly D, Swiontkowski MF, Cummings J. Comparative effectiveness of open versus minimally invasive sacroiliac joint fusion. Med Devices Evid Res. 2014;2014(7):187-193. doi:10.2147/ MDER.S60370.

29. Soriano-Baron H, Lindsey DP, RodriguezMartinez N, et al. The Effect of Implant Placement on Sacroiliac Joint Range of Motion: Posterior vs Trans-articular. Spine. 2015;40(9):E525-E530. doi:10.1097/BRS.0000000000000839.

30. Lindsey D, Perez-Orribo L, Rodriquez-

Martinez N, et al. Evaluation of a minimally invasive procedure for sacroiliac joint fusion - an in vitro biomechanical analysis of initial and cycled properties.

Med Devices Evid Res. 2014;2014(7):131-137. doi:http://dx.doi.org/10.2147/MDER.S63499. 31. Liberati A, Altman DG, Tetzlaff J, et al. The PRISMA Statement for Reporting Systematic Reviews and Meta-Analyses of Studies That Evaluate Health Care Interventions: Explanation and ElaborationPRISMA: Explanation and Elaboration. Ann Intern Med. 2009;151(4):W - 65. doi:10.7326/ 0003-4819-151-4-200908180-00136.

32. Zaidi HA, Montoure AJ, Dickman CA. Surgical and clinical efficacy of sacroiliac joint fusion: a systematic review of the literature. J Neurosurg Spine. April 2015:1-8. doi:10.3171/2014.10.SPINE14516.
33. Fairbank JC, Pynsent PB. The Oswestry Disability Index. Spine. 2000;25(22):2940-2952; discussion 2952.

34. Schroeder JE, Cunningham ME, Ross T, Boachie-Adjei O. Early Results of Sacro-Iliac Joint Fixation Following Long Fusion to the Sacrum in Adult Spine Deformity. Hosp Spec Surg J. 2013;10(1):30-35. doi:10.1007/s11420-013-9374-4. 35. Gaetani P, Miotti D, Risso A, et al. Percutaneous arthrodesis of sacro-iliac joint: a pilot study. $J$ Neurosurg Sci. 2013;57(4):297-301.

36. Mason LW, Chopra I, Mohanty K. The percutaneous stabilisation of the sacroiliac joint with hollow modular anchorage screws: a prospective outcome study. Eur Spine J. 2013;22(10):2325-2331. doi:10.1007/s00586-013-2825-2.

37. Khurana A, Guha AR, Mohanty K, Ahuja S. Percutaneous fusion of the sacroiliac joint with hollow modular anchorage screws: clinical and radiological outcome. J Bone Joint Surg Br.

2009;91(5):627-631. doi:10.1302/

0301-620X.91B5.21519.

38. Rudolf L, Capobianco R. Five-Year Clinical and Radiographic Outcomes After Minimally Invasive Sacroiliac Joint Fusion Using Triangular Implants. Open Orthop J. 2014;8:375-383. doi:10.2174/ 1874325001408010375.

39. Whang PG, Cher D, Polly D, et al. Sacroiliac Joint Fusion Using Triangular Titanium Implants vs. Non-Surgical Management: Six-Month Outcomes from a Prospective Randomized Controlled Trial. Int J Spine Surg. 2015;9(6):http://dx.doi.org/10.14444/ 2006.

40. Morris SB, DeShon RP. Combining effect size estimates in meta-analysis with repeated measures and independent-groups designs. Psychol Methods. 2002;7(1):105-125.

41. DerSimonian R, Laird N. Meta-analysis in clinical trials. Control Clin Trials. 1986;7(3):177-188.

42. R Core Team (2013). R: A Language and Environment for Statistical Computing. Vienna, Austria http://www.R-project.org/.

43. Viechtbauer W. Conducting meta-analyses in $\mathrm{R}$ with the metafor package. J Stat Softw.

2010;36(3):1-48.

44. Sachs $\mathrm{D}$, Capobianco R. Minimally invasive sacroiliac joint fusion: one-year outcomes in $40 \mathrm{pa}-$ 
tients. Adv Orthop. 2013;2013:536128. doi:10.1155/ 2013/536128.

45. Cummings J Jr, Capobianco RA. Minimally invasive sacroiliac joint fusion: one-year outcomes in 18 patients. Ann Surg Innov Res. 2013;7(1):12. doi:10.1186/1750-1164-7-12.

46. Rudolf L. MIS Fusion of the SI Joint: Does Prior Lumbar Spinal Fusion Affect Patient Outcomes? Open Orthop J. 2013 May 17;7:163-8. doi: 10.2174/ 1874325001307010163.

47. Rudolf L. Sacroiliac Joint Arthrodesis-MIS Technique with Titanium Implants: Report of the First 50 Patients and Outcomes. Open Orthop J. 2012;6:495-502. doi:10.2174/1874325001206010495.

48. Sachs D, Capobianco R. One year successful outcomes for novel sacroiliac joint arthrodesis system. Ann Surg Innov Res. 2012;6(1):13. doi:10.1186/ 1750-1164-6-13.

49. Vanaclocha VV, Verdú-López F, Sánchez-Pardo, M, et al. Minimally Invasive Sacroiliac Joint Arthrodesis: Experience in a Prospective Series with 24 Patients. J Spine. 2014;3:185. doi: 10.4172/ 2165-7939.1000185.

50. Sachs D, Capobianco R, Cher D, et al. One-year outcomes after minimally invasive sacroiliac joint fusion with a series of triangular implants: a multicenter, patient-level analysis. Med Devices Auckl NZ. 2014;7:299-304. doi:10.2147/MDER.S56491. 51. Majeed SA. Grading the outcome of pelvic fractures. J Bone Joint Surg Br. 1989;71(2):304-306. 52. Wise CL, Dall BE. Minimally invasive sacroiliac arthrodesis: outcomes of a new technique. J Spinal Disord Tech. 2008;21(8):579-584. doi:10.1097/ BSD.0b013e31815ecc4b.

53. McGuire RA, Chen Z, Donahoe K. Dual fibular allograft dowel technique for sacroiliac joint arthrodesis. Evid-Based Spine-Care J. 2012;3(3):21-28.

54. Giannikas KA, Khan AM, Karski MT, Maxwell HA. Sacroiliac joint fusion for chronic pain: a simple technique avoiding the use of metalwork. Eur Spine J. 2004;13(3):253-256. doi:10.1007/ s00586-003-0620-1.

55. Lorio MP, Polly DW Jr, Ninkovic I, Ledonio CGT, Hallas K, Andersson G. Utilization of Minimally Invasive Surgical Approach for Sacroiliac Joint Fusion in Surgeon Population of ISASS and SMISS
Membership. Open Orthop J. 2014;8:1-6. doi:10.2174/1874325001408010001.

56. Glassman SD, Copay AG, Berven SH, Polly DW, Subach BR, Carreon LY. Defining Substantial Clinical Benefit Following Lumbar Spine Arthrodesis. J Bone Jt Surg. 2008;90(9):1839-1847. doi:10.2106/JBJS.G.01095.

57. Miller L, Reckling WC, Block JE. Analysis of postmarket complaints database for the iFuse SI Joint Fusion System: a minimally invasive treatment for degenerative sacroiliitis and sacroiliac joint disruption. Med Devices Evid Res. 2013;6:77-84. doi:10.2147/MDER.S44690.

58. Cher D, Polly D, Berven S. Sacroiliac Joint pain: burden of disease. Med Devices Evid Res. 2014;7:73-81. doi:10.2147/MDER.S55197.

59. Cher DJ, Capobianco RA. Spine device clinical trials: design and sponsorship. Spine J.

2015;15(5):1133-1140. doi:10.1016/

j.spinee.2015.01.027.

\section{Corresponding Author}

Robyn Capobianco, Director, Clinical Affairs, SIBONE, Inc., 3055 Olin Avenue, Ste. 2200, San Jose, CA 95128. rcapobianco@si-bone.com

\section{Disclosures}

The funding for this systematic review was provided by SI-BONE, Inc. SI-BONE, Inc. manufactures an implant for performing MIS SI joint fusion using a lateral transarticular approach. Robyn Capobianco and Daniel Cher are employees of SI-BONE, Inc.; Jake Heiney is a consultant for SI-BONE, Inc. The authors wish to clarify that in the US, SI-BONE's iFuse Implant System is intended for sacroiliac joint fusion for conditions including sacroiliac joint dysfunction that is a direct result of sacroiliac joint disruptions and degenerative sacroiliitis. In all other countries in which the device is available commercially, the iFuse Implant System is indicated for sacroiliac joint fusion.

Published 22 July 2015.

This manuscript is generously published free of charge by ISASS, the International Society for the Advancement of Spine Surgery. Copyright @ 2015 
ISASS. To see more or order reprints or permissions, see http://ijssurgery.com. 